\title{
The Influence of Vacancy Generation at the Initial Stage of Ion Implantation
}

\author{
Elena S. Parfenova, a) and Anna G. Knyazeva, ${ }^{1,2, b)}$ \\ ${ }^{1}$ Institute of High Technology Physics, National Research Tomsk Polytechnic University, Tomsk, 634050, Russia \\ ${ }^{2}$ Institute of Strength Physics and Materials Science SB RAS, Tomsk, 634055, Russia

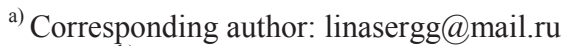 \\ b) anna-knyazeva@mail.ru
}

\begin{abstract}
The paper presents a coupled isothermal model at the initial stage of a solid surface treatment with particle beams. Mechanical stresses arising due to the interaction of particles with the surface affect the redistribution of the implanted impurity. Vacancies in the metal surface and their generation under stress are also taken into account. The kinetic law is formulated on the basis of thermodynamics of irreversible processes. The authors used numerical investigation methods. As a result, they have obtained the distributions of impurity concentration and deformations for various time moments. The authors also compare the concentration and deformation profiles with and without vacancies and study the influences of some model parameters. The effect of vacancy generation on the diffusion has been established to lead to an increase in the depth of penetration, as well as in the concentration of impurities.
\end{abstract}

Keywords: ion implantation, coupled isothermal model, mechanical stresses, diffusion, difference scheme, vacancy generation

\section{INTRODUCTION}

Material processing with charged particle fluxes makes it possible to deliberately change the state of the material surface layer, thus improving its operating abilities. It is necessary to know both the defect structure forming as a result of implantation and ion distribution. The internal surface and vacancies play a special role in the processes of diffusion transfer [1]

There are a lot of papers describing various mathematical models of such a process, but they do not give consideration to the interaction of different phenomena. For example [2, 3], study the elastic and elastoplastic waves arising due to the rapid energy input into the material but the process of ion implantation is left unaddressed. Physical phenomena associated with the vacancy generation are discussed in many papers describing diffusion in solids. Work [4] shows that radiation generates excess atoms in interstitial positions and vacancies in the area beyond the ion path. This leads to a specific spatial distribution of point defects. The removal of impurities and such defect structures from the area of the implanted layer is generally connected with elastic waves arising at the moment of introduction of accelerated ions into the solid as well as with induced static stresses in the implanted layer [5]. Thus, it is important to consider these wave processes when developing mathematical models describing the process of surface treatment, because they affect the diffusion of the implanted impurity.

The purpose of this work is to study the influence of vacancy generation on the diffusion of impurities and distribution of mechanical stress.

\section{THE MATHEMATICAL FORMULATION}

Let us use the dynamic model described in detail in [6], but it is necessary to add the kinetic equation for vacancies:

International Conference on Physical Mesomechanics of Multilevel Systems 2014

AIP Conf. Proc. 1623, 479-482 (2014); doi: 10.1063/1.4901496

(C) 2014 AIP Publishing LLC 978-0-7354-1260-6/\$30.00 


$$
\frac{\partial C}{\partial t}=-\nabla \cdot \mathbf{J}, \rho \frac{\partial^{2} \mathbf{u}}{\partial t^{2}}=\nabla \cdot \sigma, \frac{\partial C_{V}}{\partial t}=\sigma_{V}
$$

where $\mathbf{J}=-D_{0} f\left(C, C_{V}\right) \nabla C+B C \nabla \sigma_{k k}^{e}-t_{r} \mathrm{~d} \mathbf{J} / \mathrm{d} t$ is the mass flux, $\sigma$ is the component of stress tensor in the irradiation direction $(O x), t_{r}$ is the relaxation time of the mass flux, $C$ is the mass concentration of the introduced component, $\rho$ is the density of the base material; $D_{0}$ is the self-diffusion coefficient, $t$ is the time, the form of the function $f\left(C, C_{V}\right)$ depends on the "solute" structure forming during implantation, $B=D_{0} m /(R T \rho) \Delta \alpha$ is the transport coefficient under stress, $R$ is the universal gas constant, $T$ is the temperature, $m$ is the molar mass, $\Delta \alpha$ reflects the difference between concentration expansion coefficients of the introduced elements $\alpha$ and the base material $\alpha_{0}, C_{V}$ is the concentration of vacancies; $\sigma_{V}$ is the vacancy source; $\mathbf{u}$ is the displacement vector.

The stress tensor component is associated with the deformation $\varepsilon$ in the same direction by the relation:

$$
\begin{gathered}
\sigma_{i j}=\sigma_{i j}^{V}+\sigma_{i j}^{\mathrm{e}}, \\
\sigma_{i j}^{V}=-p^{V} \delta_{i j}, \quad \sigma_{i j}^{\mathrm{e}}=2 \mu \varepsilon_{i j}+\delta_{i j}\left(\lambda \varepsilon_{k k}-K w\right),
\end{gathered}
$$

where $\varepsilon_{k k}=\varepsilon_{11}+\varepsilon_{22}+\varepsilon_{33}, \quad w=3 \Delta \alpha\left(C-C_{0}\right)+3 \Delta \alpha_{V}\left(C_{V}-C_{0}\right), \lambda, \mu$ is the Lame coefficients, $K=\lambda+2 / 3 \mu$ is the elastic compression modulus; $p^{V}$ is the viscous pressure; $\sigma_{i j}^{\mathrm{e}}$ is the elastic stresses, $\Delta \alpha_{V}=\alpha_{V}-\alpha_{0}$. Tensor components of small deformations are given by the Cauchy relationships:

$$
\varepsilon_{i j}=\frac{1}{2}\left[\frac{\partial u_{i}}{\partial x_{j}}+\frac{\partial u_{j}}{\partial x_{i}}\right] .
$$

The kinetic law of vacancy generation requires special substantiation. Based on the work [6] for the entropy production and the viscous pressure in scalar processes we get the following equation:

$$
\begin{gathered}
\sigma_{V}=L_{V V} \frac{R}{m_{\mathrm{cp}}} \ln \frac{C_{V}}{C_{V}^{\mathrm{eq}}}-L_{V P} \frac{1}{T} \frac{\partial \varepsilon}{\partial t}, \\
p^{V}=L_{P V} \frac{R}{m_{\mathrm{cp}}} \ln \frac{C_{V}}{C_{V}^{\mathrm{eq}}}-L_{P P} \frac{1}{T} \frac{\partial \varepsilon}{\partial t} .
\end{gathered}
$$

For determining the values of phenomenological coefficients, special experimental studies are required. Thermodynamics suggests only that $L_{V V} \geq 0, L_{P P} \geq 0, L_{V P}=L_{P V}$ and $L_{V V} L_{P P}-L_{V P} L_{P V} \geq 0$. In this paper, their values are chosen so as to explore the qualitative nature the vacancy generation influence on the process of impurity implantation.

In the approximation of uniaxial loading $\left(\sigma_{11} \neq 0, \sigma_{22}=\sigma_{33}=0\right)$, after algebraic transformations and using the dimensionless variables $\tau=\tau / t_{*}, \xi=x / x_{*}, e=\varepsilon / \varepsilon_{*}, r^{V}=p^{V} / p_{*}^{V}$ the model becomes:

$$
\begin{gathered}
\tau_{r} \frac{\partial^{2} C}{\partial \tau^{2}}+\frac{\partial C}{\partial \tau}=\frac{\partial}{\partial \xi}\left[g\left(C, C_{V}\right) \frac{\partial C}{\partial \xi}\right]+\omega \gamma \gamma_{V} \frac{\partial}{\partial \xi}\left[C \frac{\partial C_{V}}{\partial \xi}\right]-\omega \gamma \frac{\partial}{\partial \xi}\left[C \frac{\partial e}{\partial \xi}\right], \frac{\partial^{2} e}{\partial \tau^{2}}=-\gamma \frac{\partial^{2} C}{\partial \xi^{2}}-\gamma_{V} \frac{\partial^{2} C_{V}}{\partial \xi^{2}}-k_{p} \frac{\partial^{2} r^{V}}{\partial \xi^{2}}+\frac{\partial^{2} e}{\partial \xi^{2}}, \\
\frac{\partial C_{V}}{\partial \tau}=k_{V} \ln \frac{C_{V}}{C_{V}^{e q}}-L_{V P}^{\prime} \frac{\partial e}{\partial \tau}, \quad \mathbf{J}=-g\left(C, C_{V}\right) \frac{\partial C}{\partial \xi}+C \omega \gamma\left[\frac{\partial e}{\partial \xi}-\gamma_{V} \frac{\partial C_{V}}{\partial \xi}\right]-\tau \frac{\partial \mathbf{J}}{\partial \tau}, \\
r^{V}=L_{P V}^{\prime} \ln \frac{C_{V}}{C_{V}^{\mathrm{eq}}}-L_{P P}^{\prime} \frac{\partial e}{\partial \tau}, \quad \xi=0: \mathbf{J}=\beta \phi(\tau), e=e_{0} \phi(\tau), \xi \rightarrow \infty: C=0, e=0, \\
\tau=0: C=0, e=0, \frac{\partial C}{\partial \tau}=0, \frac{\partial e}{\partial \tau}=0, C_{V}=C_{V 0},
\end{gathered}
$$

where $\tau_{r}, \omega, \gamma, \gamma_{V}, k_{p}, k_{V}, L_{V P}^{\prime}, L_{P V}^{\prime}, L_{P P}^{\prime}$ are the model parameters. 


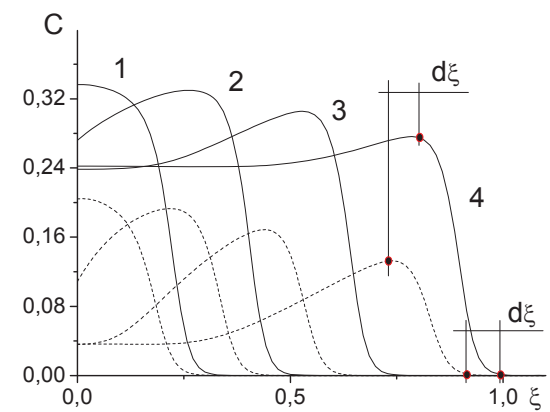

FIGURE 1. Example of solution to coupled problem with vacancies (continuous) and without vacancies (dotted), implicit scheme. Sinusoidal pulse. Times $\tau=1.57$ (1), 2.57 (2), 3.85 (3), 5.28 (4). Pulse duration $\tau_{\text {imp }}=3.0$. Initial concentration of vacancies in sample $C_{V 0}=9 \cdot 10^{-4}$

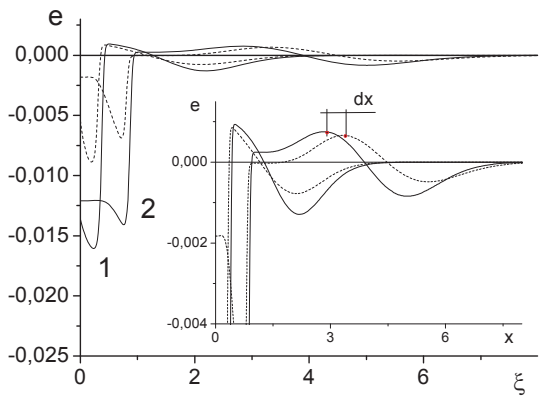

FIGURE 2. Example of solution to coupled problem with vacancies (continuous) and without vacancies (dotted), implicit scheme. Sinusoidal pulse. Times $\tau=2.57$ (1), 5.28 (2). Pulse duration $\tau_{\text {imp }}=3.0$. Initial concentration of vacancies in sample $C_{V 0}=9 \cdot 10^{-4}$

\section{RESULTS}

The model was numerically investigated using an absolutely stable implicit difference scheme. .

Figure 1 shows the distribution of the impurity concentration in depth. Initially (from the first moment of time, $\tau=1.57$ ), the effect of vacancies leads to an increase in the maximum concentration of impurities on the surface. Besides that, concentration waves are accelerated owing to the generation of vacancies. The shift of the concentration maxima is observed on the last profile $(\tau=5.28)$. Mechanical wave profiles are presented in Fig. 2 . The change of the strain sign corresponds to the penetration depth of impurities. As in the case of the impurity concentration, the generation of vacancies leads to an increase (in absolute value) in the value of deformations on the surface and acceleration of the waves.

Figure 3 shows the concentration profiles for different values of $\omega$ and $\gamma$. The reduction of $\omega$ and $\gamma$ (in absolute value) leads to an increase in the concentration wave amplitude. A slight change of the parameter $\gamma$ leads to significant changes in the concentration of impurities on the surface of the target.
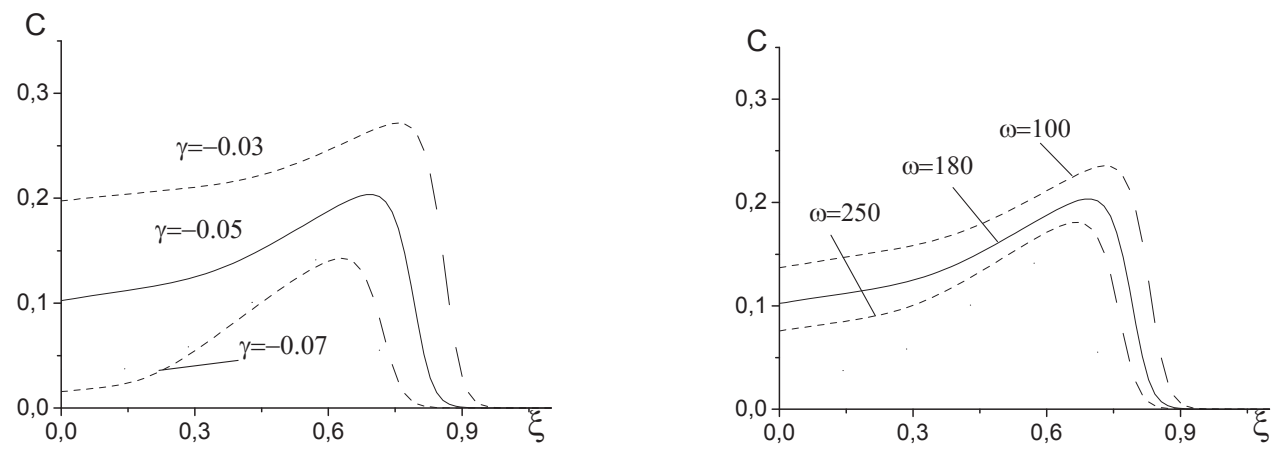

FIGURE 3. Influence of parameter $\omega=m \alpha_{0}^{2} E /(R T \rho)$ and $\gamma=\left(\alpha-\alpha_{0}\right) / \alpha_{0}$ on distribution of implanted impurity. Times, $\tau=5.28$ 
The increase of $k_{p}$ leads to a reduction in the impurity concentration. It is noteworthy that no visual difference between the diffusion profiles when $k_{p} \rightarrow 0.0 \quad\left(k^{p}=10^{-5} \ldots 10^{-\infty}\right)$ will be observed.

\section{CONCLUSION}

Thus, the paper based on the coupled isothermal model demonstrates that the interaction of the mechanical and concentration waves leads to the wave strain attenuation and its profile distortion. For the time points when the waves can be considered independent from each other, the structure of the waves is so deformed that it cannot be properly described by a purely physical mechanism of impurity transfer. The pattern becomes much more complicated as the number of pulses increases.

This work was financially supported by the Ministry of education and science of Russia for 2014 - 2016, Research No. 11.815.2014 / K.

\section{REFERENCES}

1. A. G. Knyazeva, Khim. Interes. Ustoich. Razv. 13(3), 233 (2005).

2. V. I. Boyko, Introduction to the Physics of the Interaction of Intense Beams of Charged Particles with Matter (Energoatomizdat, Moscow, 1988).

3. V. I. Boyko, Interaction of Charged Particle Beams with Matter ( Fizmatlit, Moscow, 2003).

4. A. A. Kolotov, V. Ya. Bayankin, and A. A. Novoselov, Khim. Fiz. Mezoskop. 13(3), 396 (2011).

5. A. N. Didenko, Yu. P. Sharkeev, and E. V. Kozlov, Long-Range Effects in Ion-Implanted Metallic Materials (NTL, Tomsk, 2004).

6. V. N. Demidov, A. G. Knayzeva, and E. S. Ilina, Izv. Vuzov. Fizika 5/2, 34 (2012). 